\title{
GAUSSIAN QUADRATURE FOR SUMS: A RAPIDLY CONVERGENT SUMMATION SCHEME
}

\author{
H. MONIEN
}

\begin{abstract}
Gaussian quadrature is a well-known technique for numerical integration. Recently Gaussian quadrature with respect to discrete measures corresponding to finite sums has found some new interest. In this paper we apply these ideas to infinite sums in general and give an explicit construction for the weights and abscissae of Gaussian formulas. The abscissae of the Gaussian summation have a very interesting asymptotic distribution function with a kink singularity. We apply the Gaussian summation technique to two problems which have been discussed in the literature. We find that the Gaussian summation has a very rapid convergence rate for the Hardy-Littlewood sum for a large range of parameters.
\end{abstract}

\section{INTRODUCTION}

Scalar sums of the form

$$
\sum_{n=1}^{\infty} f(n)
$$

with $n$ an integer and $f$ being a continuous function appear in nearly every context of mathematics and physics. In many problems, either because $f$ is for example the solution to another complicated nonlinear problem or there exists no known analytical expression for the sum, one has to resort to numerical summation techniques. Because of its importance many techniques have been developed for a fast evaluation of (1.1) which depend on the asymptotic behavior the function $f(x)$. A good summary of the classical techniques can be found in [2]. In the application of these schemes the sequence $s_{n}=\sum_{m=1}^{n} f(m)$ is calculated and then extrapolated to $n \rightarrow \infty$. However if the function $f$ reaches its asymptotic behavior only at very large values of $n$, then these schemes are inefficient because a large number of terms has to be accumulated before the asymptotic behavior is reached and not applicable because rounding errors prevent a reliable extrapolation. Newer convergence acceleration methods such as the Levin and Levin-Sidi transformation schemes [15, 24] have a more complicated convergence behavior and no simple statement can be made for the onset of convergence in general. The recent proposal by Strebel (see [3], chapter 3) has some very interesting properties, including very fast convergence

Received by the editor December 19, 2006 and, in revised form, October 16, 2008 and April 3, 2009.

2000 Mathematics Subject Classification. Primary 40A25; Secondary 33C90, 65D32, 33F05.

(C)2009 American Mathematical Society
Reverts to public domain 28 years from publication 
for some applications which have not been studied in detail so far. So the problem of evaluating the sum (1.1) efficiently and reliably for problems with a large intrinsic scale is interesting.

In this paper we discuss a summation scheme based on ideas related to Gauss quadrature. The basic idea is to replace the sum (1.1) by another sum

$$
\sum_{n=1}^{\infty} f(n) \approx \sum_{k=1}^{N} w_{k} f\left(x_{k}\right)
$$

where the weights $w_{k}$ and abscissae $x_{k}$ are chosen in such a way as to approximate closely the sum (1.1) for a large class of functions for a relatively small number $N$. Because the values of $x_{k}$ are not fixed to be integer numbers the hope is that the asymptotic behavior at large $x$ is captured by the sum (1.2). The central question is what are the optimal weights $w_{k}$ and points $x_{k}$ ? The answer depends of course on the function $f$, but for a certain general class of functions this question can be answered. In this paper we focus on functions $f(n)$ that are even in $n$ which are analytic in a neighborhood of $n \rightarrow \infty$.

Similar schemes have been reviewed recently by Engblom 7. Milovanović and Cvetković [19] studied the convergence properties of Gaussian summation schemes for sums of the form $\sum_{\nu>0} p^{-\nu} f\left(a q^{-\nu}\right)$, with $|p|,|q|>1$. The Gaussian quadrature using integral transforms to calculate infinite sums has been investigated by various authors [6, 13, 14, 18, 26. Uvarov and Nikiforov [22] used Chebyshev polynomials for finite sums.

\section{Moment GEnerating FUnCTIONS AND ORThOgONAL POLYNOMIALS}

Consider a real function of a complex argument $f(z)$ which has an asymptotic expansion in powers of $z^{-2}$ with a finite radius of convergence $R$,

$$
f(z)=\frac{a_{2}}{z^{2}}+\frac{a_{4}}{z^{4}}+\frac{a_{6}}{z^{6}}+\ldots
$$

for large $|z|$ with some real coefficients $a_{2}, a_{4}, a_{6}, \ldots$. The basic idea is, as in Gaussian quadrature, to expand $f(z)$ in terms of orthogonal polynomials $p_{n}$ in $k^{-2}, k \in \mathbb{N}$, and sum these exactly. The relevant scalar product is

$$
\left(p_{n} \mid p_{m}\right)=\sum_{k=1}^{\infty} \frac{1}{k^{2}} p_{n}\left(\frac{1}{k^{2}}\right) p_{m}\left(\frac{1}{k^{2}}\right) .
$$

The orthogonal polynomials could be generated by using a Gram-Schmidt orthogonalization, but in this case it is much easier to use the close connection between Padé approximants of the moment generating function and corresponding orthogonal polynomials. In fact the orthogonal polynomials corresponding to the scalar product (2.1) are given by the reverse polynomial of the denominator of the $[M-1 / M]$ Padé approximant of the moment generating function for successive values of $M$ (Baker [1, chapter 7, equation (7.7) on page 86). The moments $\sum_{k=1}^{\infty} k^{-2 m}=\zeta(2 m), m=1,2, \ldots$ are generated by

$$
\phi(z)=\sum_{m=0}^{\infty} \zeta(2 m+2) z^{m}=\frac{1}{2 z}(1-\pi \sqrt{z} \cot (\pi \sqrt{z})),
$$

which can be easily derived from the series expansion of the cot [16] (4.3.70) and the equation for the Riemann zeta function for even integers [16] (23.3.16). Using the 
continued fraction expansion of $\tan (x)$ [16] (4.3.94) we can express the generating function as:

$$
\Phi(z)=\frac{\pi^{2}}{6} \frac{1}{1-\frac{\pi^{2} z / 15}{1-\frac{\pi^{2} z / 35}{1-\frac{\pi^{2} z / 63}{1-\ldots}}}}
$$

The numerator of the $n$th Padé approximant corresponding to this continued fraction is denoted by $R_{n}(x)$ and the denominator by $S_{n}(x)$ with $x=\pi \sqrt{z}$. The denominator and numerator obey the recursion relations [2]:

$$
\begin{aligned}
R_{n+1}(x) & =R_{n}(x)+c_{n+1} x^{2} R_{n-1}(z), \\
S_{n+1}(x) & =S_{n}(x)+c_{n+1} x^{2} S_{n-1}(z),
\end{aligned}
$$

for $n=0,1,2 \ldots$ with the initial conditions for the recursion being $R_{-1}(x)=0$, $R_{0}(x)=c_{0} x, S_{-1}(x)=1$ and $S_{0}(x)=1$. The coefficients $c_{n}$ are given by $c_{0}=1 / 3$, $c_{n}=-1 /((2 n+1)(2 n+3)), n=1,2, \ldots$ This recursion generates the Padé convergents in the order $[0 / 0],[0 / 1],[1 / 1],[1 / 2], \ldots$ According to equation (7.7) in [1] the orthogonal polynomials corresponding to (2.2) are given by

$$
p_{n}(z)= \begin{cases}1 & n=0, \\ z^{n} S_{2 n-1}\left(\frac{1}{z}\right) & n \neq 0 .\end{cases}
$$

Surprisingly enough the recursion relations for $R_{n}(x)$ and $S_{n}(x)$ can be solved analytically. Let us discuss the recursion for (2.3). First note that the recursion for the Bessel functions, $Z_{\nu}$ of half-integer index $n+1 / 2$, are given by:

$$
Z_{n+3 / 2}(x)-\frac{2 n+1}{x} Z_{n+1 / 2}(x)+Z_{n-1 / 2}(x)=0 .
$$

Splitting of the asymptotic behavior for large $n$ we define $a_{n}(x)$ by requiring that

$$
Z_{n+1 / 2}(x)=\left(\frac{2}{x}\right)^{n} \Gamma\left(n+\frac{1}{2}\right) a_{n}(x) .
$$

The coefficients $a_{n}(x)$ obey the recursion relation:

$$
a_{n+1}=a_{n}-\frac{x^{2}}{4 n^{2}-1} a_{n-1} .
$$

This is precisely the recursion relation for the $S_{n}(x)$ if we shift the index $n$ by 2 . Thus the recursion relation for $S_{n}(x)$ has a solution of the form:

$$
S_{n}(x)=\frac{x^{n+2}}{2^{n+2} \Gamma\left(n+\frac{5}{2}\right)}\left[A(x) J_{n+5 / 2}(x)+B(x) Y_{n+5 / 2}(x)\right],
$$

where $A(x)$ and $B(x)$ are two unknown functions which only depend on $x$ and can be determined from the initial conditions. Specializing to the initial conditions, an explicit solution for $S_{n}(x)$ can be obtained:

$$
S_{n}(x)=-\frac{\pi x^{n+3 / 2}}{\Gamma\left(n+\frac{5}{2}\right) 2^{n+5 / 2}}\left[\cos (x) J_{n+5 / 2}(x)+\sin (x) Y_{n+5 / 2}(x)\right] .
$$


This result will be useful to determine the distribution of zeros explicitly. Using equations (2.11) and (2.6) the first few orthogonal polynomials are

$$
\begin{aligned}
p_{0}(z) & =1, \\
p_{1}(z) & =z-\frac{\pi^{2}}{15}, \\
p_{2}(z) & =z^{2}-\frac{\pi^{2}}{9} z+\frac{\pi^{4}}{945} . \\
& \ldots
\end{aligned}
$$

Using the recursion relation for the $S_{n}(z)$ it can be shown that the $p_{n}(z)$ obey the recursion relation $p_{n+1}(z)=\left(z-a_{n}\right) p_{n}(z)+b_{n} p_{n-1}(z)$ with

$$
\begin{aligned}
& a_{n}=\frac{2 \pi^{2}}{(4 n+1)(4 n+5)}, \\
& b_{n}=\frac{\pi^{4}}{(4 n-1)(4 n+1)^{2}(4 n+3)}
\end{aligned}
$$

for $n=1,2, \ldots$ and $a_{0}=\pi^{2} / 15$. These polynomials are orthogonal with respect to the following scalar product:

$$
\left(p_{n} \mid p_{m}\right)=\sum_{k=1}^{\infty} \frac{1}{k^{2}} p_{n}\left(\frac{1}{k^{2}}\right) p_{m}\left(\frac{1}{k^{2}}\right)=\delta_{n m} \frac{(4 n+3) \pi^{3}}{2 \Gamma^{2}\left(2 n+\frac{5}{2}\right) 2^{4(n+1)}},
$$

which can be derived easily from $\left(p_{n} \mid p_{n}\right)=\left(p_{0} \mid p_{0}\right) \prod_{k=1}^{n} b_{k}$ with $\left(p_{0} \mid p_{0}\right)=\pi^{2} / 6$. We note that these polynomials $p_{n}(z)$ are related to Bessel polynomials, $y_{n}(x)$, of imaginary argument [12, 22]:

$$
p_{n}(x)=\frac{\pi^{2 n+1 / 2}}{2^{2 n+1} \Gamma\left(2 n+\frac{3}{2}\right)} \operatorname{Re}\left(y_{2 n+1}\left(-i \frac{\sqrt{x}}{\pi}\right)\right)
$$

and that they appear in various contexts [17, 21].

\section{Calculation of the Weights and abscissae}

Given the three-term recursion relation for the orthogonal polynomials (2.12) the problem of finding the weights and abscissae of the Gaussian quadrature formulas for sums reduces to the standard procedure used to determine the analogous quantities in Gaussian integration [10]. The Jacobi matrix for determining the weights and abscissae is given by

$$
\tilde{J}_{N}=\left[\begin{array}{cccccc}
a_{0} & \sqrt{b_{1}} & & & & \\
\sqrt{b_{1}} & a_{1} & \sqrt{b_{2}} & & & \\
& \sqrt{b_{2}} & a_{2} & \sqrt{b_{3}} & & \\
& & \ddots & \ddots & \ddots & \\
& & & \sqrt{b_{N-2}} & a_{N-2} & \sqrt{b_{N-1}} \\
& & & & \sqrt{b_{N-1}} & a_{N-1}
\end{array}\right] .
$$

It is easy to calculate the weights and abscissae using an implementation of the Golub and Welsh algorithm [10, either "gauss.m" from the QCP collection of Gautschi 8], or the gaucof routine from the Numerical Recipes [23. Since the $p_{n}(z)$ are orthogonal polynomials in $1 / k^{2}$ the eigenvalues of (3.1) do not give the 
summation points directly but are given by $x_{k}=1 / \sqrt{\lambda_{k}}$, where $\lambda_{k}$ is the $k$ th eigenvalue of (3.1). In terms of the weights $w_{k}$ and abscissae $x_{k}$ determined from the Jacobi matrix (3.1), the Gaussian summation procedure is given by:

$$
\sum_{k=1}^{\infty} f(k)=\sum_{k=1}^{N} w_{k} f\left(x_{k}\right)+\epsilon_{N}
$$

with the standard error estimate $\epsilon_{n}$ (see e.g. 25]). The

$$
\epsilon_{N}=\frac{f^{(2 N)}(\xi)}{(2 N) !}\left(p_{N} \mid p_{N}\right),
$$

where $\xi \in \mathbb{N}$. Although the error estimate (3.3) gives an upper bound, it is not very useful to estimate the convergence of the Gaussian summation formulas. For the specific examples below we can sharpen the error estimates and achieve a more general understanding of the convergence of the Gaussian summation formulas.

\section{TWO EXAMPLES}

We apply the result of the preceding section to two examples. The first is the Hardy-Littlewood function, which has interesting properties in itself and is defined as:

$$
H(a)=\sum_{k=1}^{\infty} \frac{\sin (a / k)}{k}
$$

This summand has an expansion in terms of $1 / k^{2}$ for $k / a \gg 1$. For large values of $a$ the summation is not expected to converge before the $k \approx a$. The numerical evaluation of this function has been discussed by Gautschi [8, 9]. The methods considered in these references are Gaussian integration of the Laplace transformation of the sum and the application of Euler-MacLaurin asymptotic summation to eliminate the first asymptotic terms $O\left(1 / k^{2}\right)$ and $O\left(1 / k^{4}\right)$.

We have applied our method to problem (4.1) for $a=100$. The summation converges rapidly as shown below and for $n>14$ the error in the sum is dominated by the error in the weights and points. One observes that the differences of the value of the Gaussian summation for different $n$ is fluctuating around the machine $\epsilon$. We find that the Gaussian summation scheme achieves a high accuracy using very few function evaluations of the summand. Basically the accuracy is limited by the accuracy of the Gaussian weights and points used in the evaluation of the summation. For example, using fifteen terms, the Gaussian summation gives the result for the Hardy-Littlewood sum with an error less then $10^{-14}$ for all $a \in$ $(0,100)$. We summarize our results in Table 1 The symbol - in the table indicates when the relative error in the summation is less then $10^{-14}$. The error is plotted in Fig. 6.1 for $a=100$ as a function of $n$. Gaussian summation give results already at a relatively small number of terms which does not grow like the scale where the function reaches the asymptotic behavior.

Next we derive an analytic expression for the error estimate confirming the empirical findings. Obviously

$$
\Sigma_{n}[f]=\sum_{k=1}^{n} w_{k} f\left(x_{k}\right)=\frac{1}{2 \pi i} \int_{C} d \zeta \frac{1}{\zeta}\left(1-\frac{R_{2 n-1}(\pi \zeta)}{S_{2 n-1}(\pi \zeta)}\right) \frac{1}{\zeta^{2}} f\left(\frac{1}{\zeta^{2}}\right)
$$


where $C$ is a contour enclosing the zeros of $S_{n}(\pi \zeta)$. The error estimate $\Delta_{n}[f]=$ $\Sigma_{n+1}[f]-\Sigma_{n}[f]$ can be simplified using the recursion (2.4):

$$
\Delta_{n}[f]=\frac{1}{2 \pi i} \int_{C} d \zeta A_{n}(\pi \zeta) \frac{1}{\zeta^{2}} f\left(\frac{1}{\zeta^{2}}\right)
$$

with

$$
A_{n}(\zeta)=\frac{2 \pi(4 n+3) \pi^{4 n+1} \zeta^{4 n+1}}{2^{4 n+5} \Gamma^{2}(2 n+5 / 2)} \times \frac{1}{S_{2 n+1}(\pi \zeta) S_{2 n-1}(\pi \zeta)} .
$$

The asymptotic behavior of the error is determined by (4.3) for $n \gg 1$. Using the asymptotic form of the Bessel function on the imaginary axis [16] and introducing $\nu=2 n+5 / 2$ we obtain as the leading contribution:

$$
A_{n}(i \nu \tau) \approx 8 \nu e^{\Phi_{\nu}(\nu \tau)}
$$

with

$$
\Phi_{\nu}(z)=\sum_{ \pm}\left[-\frac{1}{2} \ln \left(\frac{z}{\nu \pm 1}\right)+\frac{1}{2} \ln \left(1+\left(\frac{z}{\nu \pm 1}\right)^{2}\right)-(\nu \pm 1) \eta\left(\frac{z}{\nu \pm 1}\right)\right]
$$

and

$$
\eta(\tau)=\sqrt{\tau^{2}+1}+\log \left(\frac{\tau}{1+\sqrt{\tau^{2}+1}}\right) .
$$

For the Hardy-Littlewood function the error can be estimated as:

$$
\Delta_{n} \approx \frac{4 \nu}{\pi} \int_{C} d \zeta \exp \left(\Phi_{\nu}(\zeta)\right) \sinh \left(\frac{\pi a}{\zeta}\right) .
$$

This integral is completely dominated by the saddle points on the imaginary axis which appear for $2 \nu^{2} /(\pi a) \gg 1$ and are located at

$$
\nu \tau_{\min }= \pm \frac{\nu}{\sqrt{\xi^{2}-2 \xi}}\left(1+\frac{\xi}{2(\xi-2) \nu}+O\left(\frac{1}{\nu^{2}}\right)\right),
$$

with the direction of steepest descent being perpendicular to the imaginary axis and $\xi$ is given by $\xi=2 \nu^{2} /(\pi a)$. The evaluation of the integral at the saddle point yields:

$$
\Delta_{n} \approx 8 \nu \sqrt{\frac{2 \pi}{\Phi_{\nu}^{\prime \prime}\left(\nu \tau_{\min }\right)}} \exp \left(\Phi_{\nu}\left(\nu \tau_{\min }\right)\right) \sinh \left(\frac{\pi a}{\nu \tau_{\min }}\right) .
$$

For $2 \nu^{2} /(\pi a) \gg 1$ this expression can be simplified further to finally yield the asymptotic error estimate:

$$
\Delta_{n} \approx 2 \sqrt{\pi \nu} e^{-\pi a / \nu}\left(\frac{e^{2} \pi a}{4 \nu^{2}}\right)^{2 \nu} .
$$

We have plotted this error estimate in Fig. 6.1. The agreement of the saddle point approximation (4.10) and the analytic asymptotic expression (4.11) with the numerical result is quite reasonable. This expression confirms that for smooth functions the Gaussian summation has a very rapid rate of convergence. The rate of convergence is determined by the scale of the function, in this case $a$ at which the asymptotic behavior sets in. However in the Gaussian summation the rate of convergence is determined by the ratio $a / \nu^{2}$ so that convergence already sets in 
at $\nu \approx \sqrt{a}$. This is a more rapid convergence rate than any of the extrapolation schemes for sums proposed in [9].

The second example serves to illustrate this point more clearly. We consider a sum known explicitly [16]:

$$
G(a)=\sum_{k=-\infty}^{\infty} \frac{1}{a^{2}+k^{2}}=\frac{\pi}{a} \operatorname{coth}(\pi a),
$$

with $a \in \mathbb{R}_{>0}$. We have applied Gaussian summation to (4.12). Again we observe rapid convergence even for values of $a$ as large as 1000. The numerical convergence pattern is completely regular. In this case the expression for the error estimator $\Delta_{n}$ can be given without approximation because the contour integral can be done exactly by deforming the contour and is given by the residues of the integrand at $\pm i a$ :

$$
\Delta_{n}=\frac{1}{2 \pi i} \int_{C} d \zeta A_{n}(\zeta) \frac{1}{a^{2}+\zeta^{2}}=\frac{A_{n}(i \pi a)}{a} .
$$

For large values of $a$, using the asymptotic expression for $A_{n}$ for $\nu^{2} \gg \pi a$ and $a \gg \nu$, this expression simplifies to:

$$
\Delta_{n} \approx 8 \nu \exp \left(-\frac{\nu^{2}}{\pi a}\right) .
$$

We contrast this behavior with other well-known techniques for acceleration of sums such as Richardson extrapolation 2. Using the Euler-MacLaurin sum formula it can be shown that the partial sums behave asymptotically as:

$$
\begin{aligned}
G_{n}(a) & =\frac{1}{a^{2}}+2 \sum_{k=1}^{n} \frac{1}{a^{2}+k^{2}} \\
& =\frac{\pi \operatorname{coth}(\pi a)}{a}-\frac{2}{n}+\frac{1}{n^{2}}+\frac{2 a^{2}-1}{3 n^{3}}-\frac{a^{2}}{n^{4}}-\frac{6 a^{4}-10 a^{2}+1}{15 n^{5}}+\cdots .
\end{aligned}
$$

The $N$ th Richardson extrapolation for the partial sums at some $n$ is given by:

$$
\mathcal{R}_{N}[G]=\sum_{k=0}^{N} \frac{(-1)^{k+N}(n+k)^{N}}{k !(N-k) !} G_{n+k}(a) .
$$

The asymptotic $k^{-2}$ behavior of the sum, (4.12), is only reached when $|k| \approx|a|$ and only then convergence of the partials sums starts. Applying Richardson extrapolation the terms in $1 / n$ are successively removed. However because the coefficients of the $n^{-m}$ terms are approaching $a^{m}$ the convergence is very slow and the asymptotic behavior only sets in when $n \gg a$. This can be seen in the plot of the error in the Richardson extrapolations of (4.12), Fig. 6.3. The relative error is given by $\Delta \mathcal{R}_{N}[G](a)=\left|\left(\mathcal{R}_{N}[G](a)-G(a)\right) / G(a)\right|$. It is clear that Gaussian summation achieves the same accuracy with a substantially smaller number of function evaluations. In fact the Richardson extrapolation shows spurious convergence for partial sums with $N \sim a$.

\section{Distribution of Zeros of the ORTHOGONAL POLYNOMials}

The distribution of zeros of the denominators of the Padé approximants $S_{n}(x)$ turns out to be quite unusual. According to (2.11) the zeros of $S_{n}(x)$ for a given 
odd integer number $n$ are determined by the equation:

$$
\cos (x) J_{n+5 / 2}(x)+\sin (x) Y_{n+5 / 2}(x)=0 .
$$

We are interested in the asymptotic behavior of the zeros at large values of $n$. Let $x>0$. The function is symmetric with respect to $x \rightarrow-x$, so for each zero $x_{0}$ there is a corresponding zero at $-x_{0}$. We define $\nu=n+5 / 2$ and $\tau=x / \nu$. The condition for a zero of the denominator apart from nonvanishing prefactors is:

$$
\cos (\nu \tau) J_{\nu}(\nu \tau)+\sin (\nu \tau) Y_{\nu}(\nu \tau)=0
$$

In the limit $\nu \rightarrow \infty$ we have to differentiate between two regimes, $\tau<1$ and $\tau>1$. For the first case, $\tau<1$, the leading asymptotic behavior of the Bessel function corresponding to $x<\nu$ is given by [16, 11]:

$$
J_{\nu}(\nu \tau)=\frac{1}{\sqrt{2 \pi \nu}} \frac{\exp \left(\nu\left(\sqrt{1-\tau^{2}}-\operatorname{arcosh}(1 / \tau)\right)\right)}{\left(1-\tau^{2}\right)^{1 / 4}}
$$

and

$$
Y_{\nu}(\nu \tau)=\frac{-2}{\sqrt{2 \pi \nu}} \frac{\exp \left(-\nu\left(\sqrt{1-\tau^{2}}-\operatorname{arcosh}(1 / \tau)\right)\right)}{\left(1-\tau^{2}\right)^{1 / 4}} .
$$

The argument of the exponent of the second term is negative in the interval $(0,1)$. The first term is exponentially suppressed compared to the second term. The zeros of the denominator for $\tau \in(0,1)$ are therefore determined by the expression:

$$
\sin (\nu \tau)=0 .
$$

From this equation we conclude that $\nu \tau=\pi m$ with $m$ an integer of the same order as $\nu$, so it is useful to introduce $\sigma=m / \nu$, so that in this regime $\tau=\pi \sigma$.

Next we consider the case $\tau>1$. The leading term in the asymptotic expansion of the Bessel functions takes a different form:

$$
\begin{aligned}
J_{\nu}(\nu \tau) & =\sqrt{\frac{2}{\pi \nu}} \cos \left(\nu\left(\sqrt{\tau^{2}-1}-\arccos (1 / \tau)\right)\right), \\
Y_{\nu}(\nu \tau) & =\sqrt{\frac{2}{\pi \nu}} \sin \left(\nu\left(\sqrt{\tau^{2}-1}-\arccos (1 / \tau)\right)\right) .
\end{aligned}
$$

For this case the leading asymptotic behavior of the zeros of the denominator have to be determined by the following equation:

$$
\cos \left(\nu\left(\tau-\sqrt{\tau^{2}-1}+\arccos (1 / \tau)\right)\right)=0 .
$$

In this regime the zeros are solutions of $\tau-\sqrt{\tau^{2}-1}+\arccos (1 / \tau)=\pi \sigma$. To summarize, the asymptotic behavior of the zeros is given by:

$$
\pi \sigma(\tau)= \begin{cases}\tau & \tau \leq 1, \\ \tau-\sqrt{\tau^{2}-1}+\arccos (1 / \tau) & \tau>1 .\end{cases}
$$

Note that $\sigma$ approaches $1 / 2$ in the limit $\tau \rightarrow \infty$. This is expected because we have considered the case $x>0$ only. In fact the distribution of zeros is symmetric in the range $\sigma \in(-1 / 2,1 / 2)$ since the $S_{n}(x)$ depend on $x^{2}$, so only half of the zeros lie above 0 . We enumerate the positive zeros by $x_{k}, k \in\{1,2, \ldots n\}$. The $n$ negative zeros are given by $-x_{k}$. The asymptotic behavior of the zeros is surprising: For large $n$ the first $[2 n / \pi]$ zeros are with high accuracy given by $x=\pi, 2 \pi, 3 \pi, \ldots$ or in terms of the original summation variable by $1,2,3 \ldots$. However for $k>[2 n / \pi]$ the distance between two consecutive zeros becomes larger. The asymptotic behavior 
has been verified in numerical determination of the zeros. The Gaussian quadrature for sums achieves its accuracy by evaluating $[2 n / \pi]$ points at the original summation points and using the rest of the points to explore the asymptotic behavior of the function which is being summed. This distribution of the zeros is unusual because a singularity appears in the range of integration which is not present in the moment generating function or the scalar product. For comparision we have plotted in Fig. 6.4 the asymptotic density of zeros for the Hermite and Laguerre polynomials as given by Calogero and Perelomov [4, 5] and the asymptotic density of zeros of $S_{n}$ as a function $2 \sigma$ to map the interval to $[-1,1]$. All densities are normalized to one. As can be clearly observed the density of zeros of the Hermite and Laguerre polynomials has a singularity at \pm 1 whereas in our case the singularity appears at $\pm \pi / 4$ and smoothly vanishes at \pm 1 .

\section{Conclusions}

The general problem of summation of smooth functions is considered. Summation formulas in analogy to Gaussian quadrature have been derived. The Padé approximants of the moment generating functions have been used to derive the explicit solution of the recursion relation. We have used this to give an explicit formula for the corresponding orthogonal polynomials. The asymptotic distribution of the zeros of these polynomials is derived. We have shown that the distribution of zeros shows an unusual behavior exhibiting a cusp. We demonstrate that Gaussian summation needs a substantially smaller number $(\sqrt{n}$ compared to $n)$ of function evaluations for smooth functions with a large scale than other common techniques. In particular higher order Richardson extrapolation will lead to rounding errors spoiling the convergence.

It would be interesting to investigate Gaussian summation for different kinds of linear functionals. Another interesting point is the development of Kronrod schemes for summation which we are currently working on.

TABLE 1. Results for the relative error in evaluation of the HardyLittlewood sum.

\begin{tabular}{|c|c|c|c|c|c|c|}
\hline$n$ & $\Delta H(1)$ & $\Delta H(5)$ & $\Delta H(10)$ & $\Delta H(20)$ & $\Delta H(40)$ & $\Delta H(100)$ \\
\hline 2 & $8.73 \cdot 10^{-9}$ & $1.58 \cdot 10^{-2}$ & $1.9 \cdot 10^{0}$ & $4.47 \cdot 10^{-1}$ & $5.06 \cdot 10^{-1}$ & $5.61 \cdot 10^{-1}$ \\
3 & - & $2.53 \cdot 10^{-6}$ & $1.02 \cdot 10^{-2}$ & $9.55 \cdot 10^{-1}$ & $2.29 \cdot 10^{-1}$ & $3.29 \cdot 10^{0}$ \\
4 & - & $3.66 \cdot 10^{-11}$ & $3.3 \cdot 10^{-6}$ & $1.67 \cdot 10^{-2}$ & $1.28 \cdot 10^{0}$ & $3.29 \cdot 10^{0}$ \\
5 & - & - & $1.47 \cdot 10^{-10}$ & $2.29 \cdot 10^{-5}$ & $2.48 \cdot 10^{-1}$ & $4.51 \cdot 10^{-1}$ \\
6 & - & - & - & $5.19 \cdot 10^{-9}$ & $3.04 \cdot 10^{-3}$ & $2.53 \cdot 10^{0}$ \\
7 & - & - & - & $2.85 \cdot 10^{-13}$ & $5.89 \cdot 10^{-6}$ & $2.77 \cdot 10^{0}$ \\
8 & - & - & - & - & $2.8 \cdot 10^{-9}$ & $1.09 \cdot 10^{0}$ \\
9 & - & - & - & - & $4.19 \cdot 10^{-13}$ & $4.46 \cdot 10^{-2}$ \\
10 & - & - & - & - & - & $3.87 \cdot 10^{-4}$ \\
11 & - & - & - & - & - & $1.02 \cdot 10^{-6}$ \\
12 & - & - & - & - & - & $1.01 \cdot 10^{-9}$ \\
13 & - & - & - & - & - & $4.07 \cdot 10^{-13}$ \\
14 & - & - & - & - & - & $2.51 \cdot 10^{-14}$ \\
15 & - & - & - & - & - & - \\
\hline
\end{tabular}




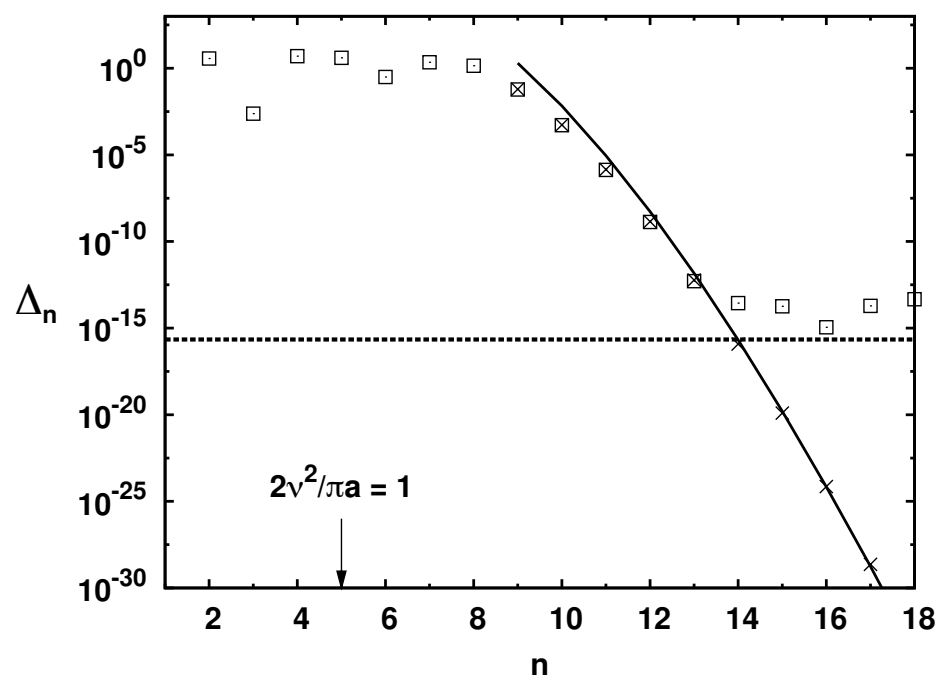

Figure 6.1. Relative error in the evaluation of the HardyLittlewood sum for $a=100$. The squares $(\square)$ are indicating the numerical error in the Gaussian summation, the crosses $(\times)$ are indicating the saddle point approximation of the error integral and the solid line gives the asymptotic behavior of the former for large $n$. The dashed line gives the machine $\epsilon$ for comparison.

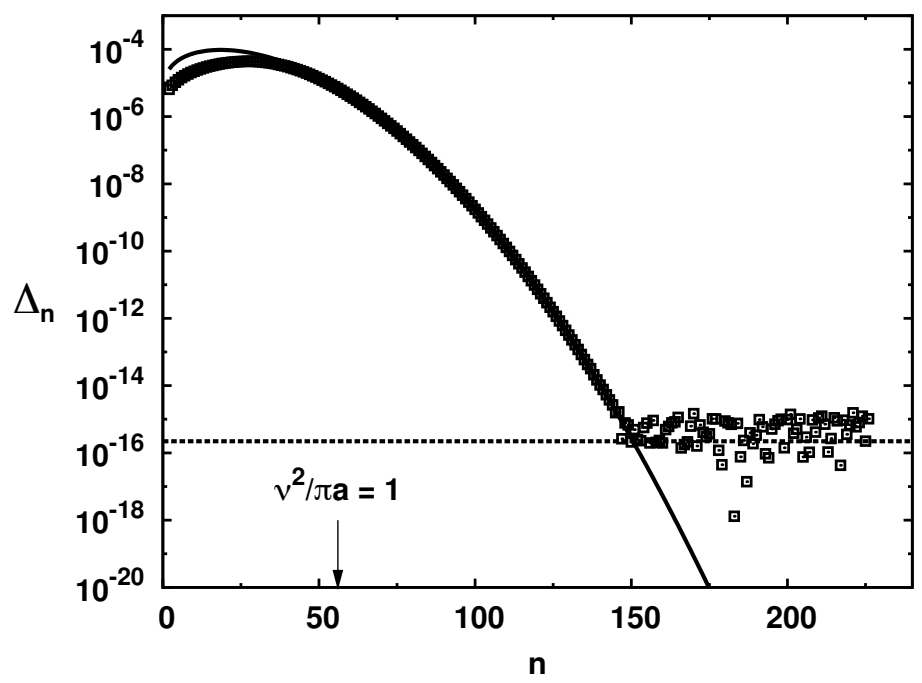

Figure 6.2. Relative error of the Gaussian summations of example 2, sum (4.12), for $a=1000$. The solid line is the error estimate given in the text $\left(\sim 8 n \exp \left(-4 n^{2} /(\pi a)\right)\right)$ and the dotted line is giving the machine $\epsilon$. 


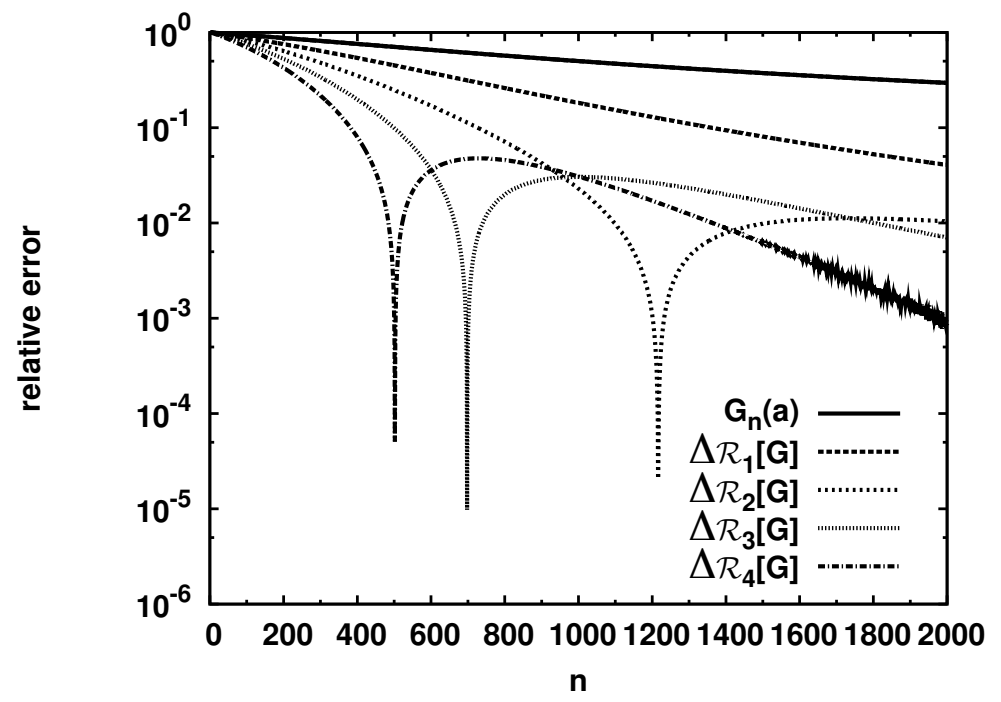

FiguRe 6.3. Relative error of the Richardson extrapolations of example 2, sum (4.12), for $a=1000$. Note the appearance of rounding errors in $\Delta \mathcal{R}_{4}[G](n)$.

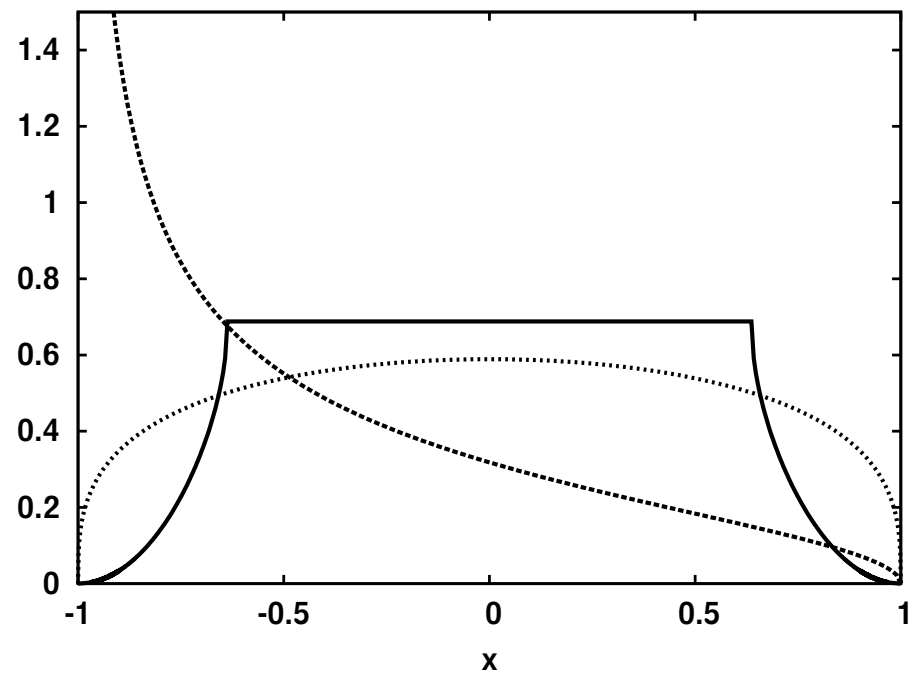

Figure 6.4. Asymptotic density of zeros of the polynomials $S_{n}$ (solid line), the Hermite polynomials (dotted line) and the Laguerre polynomials (dashed line). Note that the density of the Hermite and Laguerre polynomials has a singular behavior at $x= \pm 1$ whereas the density of zeros of the $S_{n}$ has a kink singularity at $x= \pm \pi / 4$. 


\section{Acknowledgments}

I would like to acknowledge useful discussions with Stefan Engblom and thank him in particular for writing his technical report [7] on this interesting subject and providing me with reference [20]. During the initial stage of this work I obtained useful comments from W. Gautschi which were greatly appreciated. I would like to thank Simon Trebst and Gang Li for carefully reading the manuscript. Finally I would like to thank Andrew J. Millis for useful discussions and his remark, "If you want a number, then calculate." I would like to thank the Aspen Center for Physics, where part of this work was done.

\section{REFERENCES}

1. George A. Baker, Jr., Essentials of Padé Approximants, Academic Press, NY, 1975. MR0454459 (56:12710)

2. Carl M. Bender and Steven A. Orzag, Advanced Mathematical Methods for Scientists and Engineers, McGraw-Hill, 1978 MR0538168 (80d:00030)

3. Folkmar Bornemann, Dirk Laurie, Stan Wagon, and Jörg Waldvogel, The SIAM 100digit challenge: A study in high-accuracy numerical computing, SIAM, 2004. MR2076374 (2005c:65002)

4. F. Calogero and A. M. Perelomov, Asymptotic Density of the Zeros of Hermite Polynomials of Diverging Order, and Related Properties of Certain Singular Integral Operators, Lettere al Nuovo Cimento 23 (1978), no. 18, 650-652. MR.522774 (80f:33015)

5. _ Asymptotic Density of the Zeros of Laguerre and Jacobi Polynomials, Lettere al Nuovo Cimento 23 (1978), no. 18, 653-656. MR522775 (80f:33016)

6. Germund Dahlquist, Summation formulas due to Plana, Lindelöf and Abel, and related Gauss-Christoffel rules I, BIT 37 (1997), no. 2, 256-295. MR1450961 (99a:65010)

7. Stefan Engblom, Gaussian quadratures with respect to discrete measures, Uppsala University, Technical Report 2006-007 (2006), 1-17.

8. Walter Gautschi, Orthogonal Polynomials, Oxford University Press, 2004. MR2061539 (2005e:42001)

9. , The Hardy-Littlewood Function: An Exercise in Slowly Convergent Series, J. Comput. Appl. Math. 179 (2005), 249-254. MR2134369 (2006d:33002)

10. G. H. Golub and J. H. Welsh, Calculation of Gauss quadrature rules, Math. Comp. 23 (1969), 221-230. MR0245201 (39:6513)

11. I. S. Gradshteyn and I. M. Ryzhik, Table of Integrals, Series and Products, Academic Press, 1994. MR1243179 (94g:00008)

12. Emil Grosswald, Bessel polynomials, Lecture Notes in Mathematics, Springer, 1978. MR520397 (80i:33013)

13. Sven-Ake Gustafson, Convergence acceleration by means of numerical quadrature, BIT 6 (1966), 117-128. MR0205430(34:5258)

14. _ A method of computing limit values, Siam J. Numer. Anal. 10 (1973), no. 6, 10801090. MR0343528 (49:8269)

15. D. Levin, Methods for accelerating convergence of infinite series and integrals, Ph.D. thesis, Tel Aviv University, 1975.

16. M. Abramowitz and Irene A. Stegun (ed.), Handbook of Mathematical Functions, Dover, 1965.

17. Gradimir V. Milovanović, Various extremal problems of Markov's type for algebraic polynomials, Facta Universitatis (Nis) 2 (1987), 7-28. MR963780 (90c:41010)

18. Summation of Series and Gaussian Quadratures, Approximation and Computation ISNM 119 (1994), 459-475. MR.1333636 (96c:65041)

19. Gradimir V. Milovanović and Aleksandar S. Cvetković, An application of little 1/q-Jacobi polynomials to summation of certain series, Facta Univ. Ser. Math. Inform. 18 (2003), 31-46. MR2027228 (2005b:33012)

20. - Convergence of Gaussian Quadrature Rules for Approximation of Certain Sums, Eastern J. on Approximation 10 (2004), 171-187. MR2074906 (2005e:41079) 
21. Gradimir V. Milovanović, D. S. Mitrivonić, and Th. M. Rassias, Topics in polynomials: Extremal problems, inequalities, zeros, World Scientific, Singapore, New Jersey, London and Hong Kong, 1994. MR.1298187 (95m:30009)

22. Arnold F. Nikiforov and Vasilii B. Uvarov, Special Functions of Mathematical Physics, Birkhäuser Verlag, Basel, 1978. MR0538275 (81b:33001)

23. William H. Press, Saul A. Teukolsky, William T. Vetterling, and Brian P. Flannery, Numerical Recipes in $C++$, Cambridge University Press, 2002. MR.1880993 (2003a:65005)

24. A. Sidi, Practical Extrapolation Methods: Theory and Applications, Cambridge University Press, 2003. MR 1994507 (2004e:65005)

25. J. Stoer and R. Burlisch, Introduction to numerical analysis (3rd ed.), Springer, 2002. MR 1923481 (2003d:65001)

26. W. Gautschi and Gradimir V. Milovanović, Gaussian Quadrature Involving Einstein and Fermi Functions with an Application to Summation of Series, Math. Comp. 44 (1985), no. 169, 177-190. MR771039 (86j:65028)

Bethe Center for Theoretical Physics, Universität Bonn, Nussallee 12, 53115 Bonn, GERMANY

E-mail address: monien@th.physik.uni-bonn.de 